\title{
CO observations of water-maser post-asymptotic giant branch stars and detection of a high-velocity outflow in IRAS 15452-5459
}

\author{
L. Cerrigone ${ }^{1}$, K. M. Menten ${ }^{2}$, and T. Kamiński ${ }^{2}$
}

${ }^{1}$ Centro de Astrobiología, INTA-CSIC, ctra de Ajalvir km 4, 28850 Torrejón de Ardoz, Spain
e-mail: cerrigone@cab.inta-csic.es
2 Max-Planck-Institut für Radioastronomie (MPIfR), Auf dem Hügel 69, 53121 Bonn, Germany

Received 19 December 2011 / Accepted 21 March 2012

\section{ABSTRACT}

\begin{abstract}
Many aspects of the evolutionary phase in which asymptotic giant branch stars (AGB stars) are in transition to become planetary nebulae $(\mathrm{PNe})$ remain poorly understood. An important question is how the circumstellar envelopes of AGB stars switch from spherical symmetry to the axially symmetric structures frequently observed in PNe. In many cases, there is clear evidence that the shaping of the circumstellar envelopes of PNe is linked to the formation of jets/collimated winds and their interaction with the remnant AGB envelope. Because of the short timescale of this evolutionary phase, objects are rare, but their identification provides valuable probes for testing evolutionary models. We observed (sub)millimeter CO rotational transitions with the APEX telescope in a small sample of stars hosting high-velocity $\mathrm{OH}$ and water masers. These targets are assumed to have recently left the AGB, as indicated by the presence of winds traced by masers with velocities larger than observed during that phase. We carried out observations of several CO lines, ranging from $J=2-1$ up to $J=7-6$. In IRAS $15452-5459$, we detect a fast molecular outflow in the central region of the nebula and estimate a mass-loss rate between $1.2 \times 10^{-4} M_{\odot} \mathrm{yr}^{-1}$ (assuming optically thin emission) and $4.9 \times 10^{-4} M_{\odot} \mathrm{yr}^{-1}$ (optically thick emission). We model the SED of this target taking advantage of our continuum measurement at $345 \mathrm{GHz}$ to constrain the emission at long wavelengths. For a distance of $2.5 \mathrm{kpc}$, we estimate a luminosity of $8000 L_{\odot}$ and a dust mass of $\sim 0.01 M_{\odot}$. Through the flux in the [CII] line $(158 \mu \mathrm{m})$, we calculate a total mass of about $12 M_{\odot}$ for the circumstellar envelope, but the line is likely affected by interstellar contamination.
\end{abstract}

Key words. stars: AGB and post-AGB - circumstellar matter - submillimeter: stars

\section{Introduction}

When a low- or intermediate-mass star has completed its core helium burning, it goes through the asymptotic giant branch (AGB) phase, which is characterized by heavy mass-loss. Dust formation is very efficient in AGB outflows and about 1-2\% of the mass of the envelope is condensed into dust grains. After leaving the AGB, the mass-loss rate drops substantially and the star may become optically visible, as its dusty shell disperses. Eventually, once it reaches a temperature of 20000-30000 K, the central star will start to ionize its circumstellar envelope (CSE) and a planetary nebula (PN) will form (Habing 1996). Dusty CSEs re-radiate the absorbed stellar light and show clear signatures in the far-infrared (FIR) spectrum. In particular, a "color-color" diagram introduced by van der Veen \& Habing (1988) has permitted a classification of sources to be developed based on the ratios of flux densities measured with the InfraRed Astronomical Satellite (IRAS) .

The heavy mass-loss of AGB stars (with rates of up to several $10^{-4} M_{\odot} \mathrm{yr}^{-1}$ ) is one of the most important pathways for mass return from stars to the interstellar medium (ISM).

After the AGB phase, the star "somehow" develops a less massive $\left(10^{-7} M_{\odot} \mathrm{yr}^{-1}\right)$ but faster (up to $10^{-3} \mathrm{~km} \mathrm{~s}^{-1}$ ) wind that sweeps up the circumstellar material, possibly creating shocks and high-density shells. Ultraviolet (UV) photons produced at this higher stellar temperature can excite, ionize, and dissociate molecules, heat the dust, and ionize the gas (when $\left.T_{\text {eff }}>20-30 \times 10^{3} \mathrm{~K}\right)$.
In the CSEs of many O-rich AGB stars, maser emission is detected from the $\mathrm{SiO}, \mathrm{H}_{2} \mathrm{O}$, and $\mathrm{OH}$ molecules. The conditions for the formation of maser emission from these different molecules are typically met in a progression of zones. The $\mathrm{SiO}$ masers arise from the closest regions to the central star, and those of $\mathrm{H}_{2} \mathrm{O}$ and $\mathrm{OH}$ at progressively farther distances. In stars with thicker envelopes, longer periods, and higher mass-loss rates - the socalled $\mathrm{OH} / \mathrm{IR}$ stars - the spectral profiles of $\mathrm{H}_{2} \mathrm{O}$ and $\mathrm{OH}$ masers are often double-peaked, and the velocity range covered by the $\mathrm{OH}\left(10-25 \mathrm{~km} \mathrm{~s}^{-1}\right)$ is usually larger than that of the $\mathrm{H}_{2} \mathrm{O}$ feature (Engels 2002). When the mass-loss process ends (i.e., at the end of the AGB) and the circumstellar envelope is progressively diluted, the maser lines may start to disappear. In a spherically symmetric CSE, the $\mathrm{SiO}$ lines are the first to vanish, then those of $\mathrm{H}_{2} \mathrm{O}$, and finally those of $\mathrm{OH}$ (Lewis 1998). Given these considerations, OH/IR stars with detached circumstellar shells, whose presence can be inferred from either doubly or multiply peaked $\mathrm{OH}$-maser spectra with a large velocity spread, are good candidate objects for studying the post-AGB phase.

The members of a small but growing sub-class of water-maser stars have been dubbed "water-fountain" nebulae and show unique spectral and spatial characteristics (Likkel \& Morris 1988). The velocity spread of their $\mathrm{H}_{2} \mathrm{O}$ spectra is very large, up to several $100 \mathrm{~km} \mathrm{~s}^{-1}$, and frequently larger than that of their $\mathrm{OH}$ spectra. High-resolution interferometric observations have shown that their masers are in collimated jets with dynamical ages younger than $150 \mathrm{yr}$ (Boboltz \& Marvel 2005). Water-maser post-AGB stars and particularly water fountains 
represent a very short evolutionary phase right after the end of the AGB; they therefore provide a unique opportunity for studying the physical conditions in the molecular envelopes as the shaping agent begins to act.

One such star is IRAS 15452-5459, which appears to display evidence of $\mathrm{SiO}, \mathrm{H}_{2} \mathrm{O}$, and $\mathrm{OH}$ masers. It was first selected by van der Veen et al. (1989) within a sample of sources detected with IRAS as a candidate star in transition from the AGB to the PN phase. They required that their targets fall within regions IIIb, IV, and V of the van der Veen \& Habing IRAS color-color diagram (i.e., an evolutionary sequence from the AGB to the PN), thus be bright at $12 \mu \mathrm{m}$ ( $>2 \mathrm{Jy}$ ) and have a small IRAS variability index $(<50)$. IRAS $15452-5459$ (hereafter I15452) falls within region IV of that diagram, where variable stars with very thick O-rich circumstellar shells are expected. The authors assumed a stellar temperature of $10^{4} \mathrm{~K}$ and a luminosity of $1.5 \times 10^{4} L_{\odot}$, estimating a distance of about $2.5 \mathrm{kpc}$.

Oudmaijer et al. (1995) performed IR $K$-band spectral observations and found CO ro-vibrational lines in absorption, which made them conclude that the central star should have a temperature in the range of $3000-5000 \mathrm{~K}$.

This lower stellar temperature appears to be more consistent with the CSE of I15452 indeed hosting masers in all the species typically found in CSEs. The object was included in the survey of OH masers carried out by te Lintel Hekkert et al. (1991), who detected a peculiar $\mathrm{OH}$ maser at $1612 \mathrm{MHz}$ with four intensity peaks. The features span roughly -20 to $-100 \mathrm{~km} \mathrm{~s}^{-1}$ and their peaks are found around $-83,-67,-47$, and $-33 \mathrm{~km} \mathrm{~s}^{-1}$, with a central velocity around $-57 \mathrm{~km} \mathrm{~s}^{-1}$. Subsequent observations with the Australia Telescope Compact Array confirmed the detection of the components at larger velocities and their association with the star (Deacon et al. 2004).

Deacon et al. (2007) detected the $\mathrm{H}_{2} \mathrm{O}$ maser line at $22 \mathrm{GHz}$. They found an irregular spectrum with many emission features, covering roughly -30 to $-80 \mathrm{~km} \mathrm{~s}^{-1}$ and pointing to a complex masing region. They carried out three runs of observations over one year and concluded that the spectral features are stable in velocity over a timescale of a few months, but the peak intensity of the individual features and the integrated spectral flux density vary dramatically $(\sim 70 \%)$. They also report the detection of an $\mathrm{SiO}$ maser at $86 \mathrm{GHz}$ with an unusual doubly peaked profile, with features centered on $\sim-40$ and $\sim-75 \mathrm{~km} \mathrm{~s}^{-1}$. The detection of $\mathrm{OH}, \mathrm{H}_{2} \mathrm{O}$, and $\mathrm{SiO}$ masers and the near-IR $\mathrm{CO}$ absorption indicate that the source is an O-rich star that has very recently evolved off the $\mathrm{AGB}$ and started its transition across the HR diagram.

\section{Observations and results}

\subsection{IRAS 15452-5459}

We observed I15452 with the APEX 12 m telescope, which is located at an altitude of $5100 \mathrm{~m}$ on Llano de Chajnantor, in Chile (Güsten et al. 2006). Observations were performed in both continuum and spectral-line mode, to investigate its CSE dusty and molecular components.

\footnotetext{
1 This publication is based on data acquired with the Atacama Pathfinder EXperiment (APEX). APEX is a collaboration between the Max-Planck-Institut für Radioastronomie, the European Southern Observatory, and the Onsala Space Observatory.
}

\subsubsection{CO observations}

The CO observations were carried out on 2009 October 16 and 19. The receivers APEX-2 and FLASH (Riscacher et al. 2006; Heyminck et al. 2006) were used to observe the CO $J=$ $3-2(345.796 \mathrm{GHz})$ and $J=4-3(461.041 \mathrm{GHz})$ rotational lines, respectively. The fast Fourier transform spectrometer (FFTS) backend available at APEX allows for a spectral coverage of about $1.8 \mathrm{GHz}$, split up into 8192 channels, which gives velocity resolutions of 0.21 and $0.12 \mathrm{~km} \mathrm{~s}^{-1}$ at 345 and $460 \mathrm{GHz}$, respectively (Klein et al. 2006).

Since the source is known from infrared imaging to be extended over about $25^{\prime \prime}$, we pointed the telescope at three different positions. One pointing was performed at the central coordinates of the source (right ascension 15:49:11.38; declination $-55: 08: 51.6$ ), one was offset by $+7^{\prime \prime}$ in both RA and Dec (this is indicated in the following as the north-eastern pointing) and a third pointing was similarly offset by $-7^{\prime \prime}$ (indicated in the following as the south-western pointing); each offset pointing was therefore about $10^{\prime \prime}$ away from the central coordinates.

The observations were carried out using a position-switching mode, with the reference position located $1^{\circ}$ away in azimuth. The data reduction consisted in averaging together the scans performed for each pointing and removing a low-order $(\leq 2)$ polynomial baseline. To enhance the signal-to-noise ratio, the data were smoothed to resolutions of $0.63 \mathrm{~km} \mathrm{~s}^{-1}$ for the $J=3-2$ line and $2.4 \mathrm{~km} \mathrm{~s}^{-1}$ for the $J=4-3$ line. The raw spectra are stored in the $T_{\mathrm{A}}^{\star}$ scale and can be converted to main-beam brightness temperature using $T_{\mathrm{mb}}=T_{\mathrm{A}}^{\star} \frac{F_{\text {eff }}}{B_{\text {eff }}}$, where $T_{\mathrm{A}}^{\star}$ is the antenna temperature corrected for atmospheric attenuation using the chopperwheel method, $F_{\text {eff }}$ is the telescope forward efficiency (for APEX $F_{\text {eff }} \sim 0.95$ at $345 \mathrm{GHz}$ and 0.97 at $460 \mathrm{GHz}$ ), and $B_{\text {eff }}$ is the main-beam efficiency, estimated to be 0.73 at $345 \mathrm{GHz}$ and 0.61 at $460 \mathrm{GHz}$ (Güsten et al. 2006). The uncertainty in the absolute intensity scale is estimated to be about $\pm 20 \%$. Regular pointing checks were made on strong circumstellar $\mathrm{CO}$ sources and typically found to be consistent to within $\sim 3^{\prime \prime}$. The full width at half maximum (FWHM) of the main beam, $\theta_{\mathrm{mb}}$, is $17.3^{\prime \prime}$ at $345 \mathrm{GHz}$ and $13.3^{\prime \prime}$ at $460 \mathrm{GHz}$. Table 1 summarizes the integration times, system temperatures, and rms noise for each pointing.

\subsubsection{Continuum observations}

On 2007 May 28, we observed I15452 with the Large APEX BOlometer CAmera (LABOCA) at $870 \mu \mathrm{m}(345 \mathrm{GHz})$. LABOCA is an array of 295 bolometers, operating in totalpower mode at a temperature of $\sim 285 \mathrm{mK}$. The noise-weighted mean point-source sensitivity of the array determined from onsky integrations is $55 \mathrm{mJy} \mathrm{s}^{1 / 2}$ per channel (Siringo et al. 2009).

The absolute calibration is achieved by observations of planets, namely Mars, Uranus, and Neptune. The overall calibration accuracy for LABOCA is about $10 \%$ (Siringo et al. 2009).

A major issue in performing sub-mm observations is the atmospheric opacity. Two independent methods are typically used to determine the sky opacity at APEX. The first one relies on the measurement of precipitable water vapor performed every minute by the APEX radiometer along the line of sight (Pardo et al. 2001). This approach is limited by the knowledge of the passband, the applicability of the atmospheric model, and the accuracy of the radiometer. The second method uses skydips, i.e. measurements of the power of the atmospheric emission as a function of the airmass. The values of the sky opacity resulting from the skydip analysis are robust, though up to $\sim 30 \%$ larger than those obtained by the radiometer. This systematic difference 
Table 1. Summary of the observations of IRAS $15452-5459$ performed on 2009 October $16(J=3-2)$ and 2009 October $19(J=4-3)$.

\begin{tabular}{lcccccc}
\hline \hline Pointing & \multicolumn{3}{c}{$J=3-2$} & \multicolumn{3}{c}{$J=4-3$} \\
& $\begin{array}{c}\text { Time } \\
\text { min }\end{array}$ & $\begin{array}{c}T_{\text {sys }} \\
\text { K }\end{array}$ & $\begin{array}{ccccc}\text { rms } \\
\text { mK }\end{array}$ & $\begin{array}{c}\text { Time } \\
\text { min }\end{array}$ & $\begin{array}{c}T_{\text {sys }} \\
\text { K }\end{array}$ & $\begin{array}{c}\text { rms } \\
\text { mK }\end{array}$ \\
\hline Northeast $^{a}$ & 2 & 444 & 125 & 8 & 847 & 89 \\
Center & 3.3 & 460 & 87 & 8 & 776 & 83 \\
Southwest $^{b}$ & 0.7 & 444 & 213 & 8 & 798 & 80 \\
\hline
\end{tabular}

Notes. The rms noise is calculated after smoothing the spectra to 0.63 and $2.4 \mathrm{~km} \mathrm{~s}^{-1}$ for the $J=3-2$ and $J=4-3$ line, respectively. ${ }^{(a)}$ The north-east pointing was offset by 7" in RA and 7" in Dec. ${ }^{(b)}$ The south-west pointing was offset by -7 " in RA and -7 " in Dec.

Table 2. Characteristics of $\mathrm{CO}$ observations in water fountain sources.

\begin{tabular}{|c|c|c|c|c|c|c|c|}
\hline \multirow[t]{2}{*}{ IRAS ID } & \multirow[t]{2}{*}{$\mathrm{OH}$} & \multicolumn{2}{|c|}{ Coordinates } & \multirow{2}{*}{$\begin{array}{c}V_{\mathrm{LSR}^{a}} \\
\left(\mathrm{~km} \mathrm{~s}^{-1}\right)\end{array}$} & \multirow{2}{*}{$\begin{array}{c}\text { Date } \\
\text { of observation }\end{array}$} & \multirow{2}{*}{$\begin{array}{c}\mathrm{CO} \\
\text { transition } \\
\end{array}$} & \multirow{2}{*}{$\begin{array}{r}\mathrm{rms}^{b} \\
\mathrm{mK} \\
\end{array}$} \\
\hline & & $\alpha_{\mathrm{J} 2000}$ & $\delta_{\mathrm{J} 2000}$ & & & & \\
\hline \multirow[t]{4}{*}{$18327-0715$} & $024.692+00.235^{c}$ & $18: 35: 29.82$ & $-07: 13: 09.4$ & 42.0 & 2010-07-06 & $2-1$ & 77 \\
\hline & & & & & 2010-07-07 & $3-2$ & 19 \\
\hline & & & & & 2010-07-09 & $6-5$ & 48 \\
\hline & & & & & 2010-07-09 & $7-6$ & 165 \\
\hline \multirow[t]{4}{*}{$18043-2116$} & $009.097-00.392^{c}$ & $18: 07: 21.20$ & $-21: 16: 14.0$ & 87.3 & 2010-07-05,06 & $2-1$ & 79 \\
\hline & & & & & 2010-07-07 & $3-2$ & 26 \\
\hline & & & & & 2010-07-09 & $6-5$ & 91 \\
\hline & & & & & 2010-07-09 & $7-6$ & 298 \\
\hline \multirow[t]{2}{*}{$15103-5754$} & $320.9063-00.2929^{d}$ & $15: 14: 18.47$ & $-58: 05: 20.9$ & -47.0 & 2010-07-06 & $2-1$ & 71 \\
\hline & & & & & 2010-07-07 & $3-2$ & 22 \\
\hline \multirow[t]{2}{*}{$19190+1102$} & $046.2559-01.4764^{d}$ & $19: 21: 25.30$ & $+11: 08: 40.0$ & 50.0 & 2010-07-06 & $2-1$ & 53 \\
\hline & & & & & 2010-07-07 & $3-2$ & 25 \\
\hline \multirow[t]{2}{*}{-} & $012.816-00.895^{c}$ & $18: 16: 49.23$ & $-18: 15: 01.8$ & -55.8 & 2010-07-05,06 & $2-1$ & 25 \\
\hline & & & & & 2010-07-07 & $3-2$ & 23 \\
\hline $18103-1738$ & $012.973+00.133^{c}$ & $18: 13: 20.24$ & $-17: 37: 17.3$ & 17.0 & $2010-11-16$ & $2-1$ & 35 \\
\hline
\end{tabular}

Notes. ${ }^{(a)}$ Object LSR radial velocity from Deacon et al. (2004) and Deacon et al. (2007). ${ }^{(b)}$ Noise rms level of the spectrum given per bin of $1 \mathrm{~km} \mathrm{~s}^{-1}$ and in the $T_{\mathrm{mb}}$ units. ${ }^{(c)}$ From Sevenster (2002) ${ }^{(d)}$ From SIMBAD (http://simbad.u-strasbg. fr/simbad/).

is not well-understood, therefore to reconcile the results obtained with the two methods, a linear combination of radiometer and skydip values was used, so as to obtain the most consistent calibrator fluxes at all elevations, as indicated by Siringo et al. (2009).

The observations were carried out in raster-spiral mapping mode. In the basic spiral mode, spiral scans are performed at a constant angular speed and an increasing radius around the pointing position, producing fully sampled maps of the whole field of view. These spirals are the preferred observing mode for sources brighter than a few Jy. For fainter sources, the basic spiral pattern can be combined with a raster mapping mode (rasterspirals) on a grid of pointing positions resulting in a denser sampling of the maps and a longer integration time (Siringo et al. 2009).

The data reduction was performed with the Bolometer array data Analysis (BoA) software, specifically developed for APEX bolometer data. BoA allows the observer to perform all of the necessary reduction steps: opacity correction, flux calibration, bad channel flagging, removal of correlated noise, despiking, data weighting, creation of a map, and image analysis (Siringo et al. 2009).

The nebula shows a flux density at $345 \mathrm{GHz}$ of $260 \pm 40 \mathrm{mJy}$, where the error includes the rms noise and the absolute calibration uncertainty.

\section{2. $\mathrm{CO}$ in water-maser stars}

Using the APEX telescope, we made an attempt to find $\mathrm{CO}$ rotational emission in six selected objects known to host highvelocity water masers, such as I15452. The sample included four water-fountain nebulae toward which no $\mathrm{CO}$ emission had previously been reported. All targets are listed in Table 2. The six sources were observed in the $\mathrm{CO}(2-1)$ transitions with the APEX-1 receiver, five of those were also observed in the $\mathrm{CO}(3-2)$ transitions with the APEX-2 receiver, and two of them were also observed with the CHAMP+ array (Kasemann et al. 2006) producing spectra centered on the $\mathrm{CO}(6-5)$ and (7-6) lines. The observing dates and noise levels are given in Table 2. The telescope beam widths and main beam efficiencies at the observed frequencies are given in Table 3. As a backend, we used the digital FFTS, which provided spectra at resolutions between $0.2-0.6 \mathrm{~km} \mathrm{~s}^{-1}$ and bandwidths of at least several hundred $\mathrm{km} \mathrm{s}^{-1}$.

The stars were selected from currently available maser surveys (Deacon et al. 2004; Deacon et al. 2007) as those that have both doubly peaked maser profiles with velocities larger than typical AGB values (10-25 $\mathrm{km} \mathrm{s}^{-1}$ ) and IRAS colors typical of post-AGB stars, and that are observable from the APEX site. All of them host $\mathrm{H}_{2} \mathrm{O}$ masers and are assumed to have just reached their post-AGB evolution. Imai et al. (2009) observed 9 of the 13 water fountains currently known in the CO $J=3-2$ line 
Table 3. Telescope specifications for the observed frequencies.

\begin{tabular}{lccc}
\hline \hline $\begin{array}{l}\mathrm{CO} \\
\text { transition }\end{array}$ & $\begin{array}{c}\text { Frequency } \\
(\mathrm{GHz})\end{array}$ & $\begin{array}{c}\mathrm{Beam}^{a} \\
(\operatorname{arcsec})\end{array}$ & $\eta_{\mathrm{mb}}{ }^{b}$ \\
\hline $2-1$ & 230.538 & 27 & 0.75 \\
$3-2$ & 345.796 & 17.3 & 0.73 \\
$4-3$ & 461.041 & 13.3 & 0.61 \\
$6-5$ & 691.473 & 9 & 0.56 \\
$7-6$ & 806.652 & 7.7 & 0.43 \\
\hline
\end{tabular}

Notes. ${ }^{(a)}$ Half-power beam width. ${ }^{(b)}$ Antenna main beam efficiency.

with the Atacama Submillimeter Telescope Experiment (ASTE). Their rms noise ranged from 4 to $62 \mathrm{mK}$ and they detected the line in two targets. In our selection, we excluded the water fountains observed by Imai et al. (2009) and included the newly discovered water fountain IRAS $19190+1102$ (Day et al. 2010). This leaves us with four water fountains and an additional two stars with water masers.

The observations in the $\mathrm{CO}(2-1)$ and $\mathrm{CO}(3-2)$ lines were obtained in position switching (total power) mode. Many of the sources that we observed are located close to the Galactic plane, therefore their reference positions were chosen $1-3^{\circ}$ away from the Galactic plane, to avoid emission at the OFF positions. All the observed lines of sight cross interstellar molecular clouds, which give rise to prominent emission features that are particularly strong in the $\mathrm{CO}(2-1)$ line. For all sources other than $\mathrm{OH}$ 12.8-0.9, these interstellar emission features occur in the range of velocities where the maser emission is observed. This strongly hampers the identification of any potentially existing $\mathrm{CO}$ emission from the circumstellar material in our objects. For a majority of the objects, strong interstellar lines overlap in spectral regions close to the expected source velocity. For IRAS 18103-1738, we find one feature that is centered on the stellar velocity, when we compare the $\mathrm{CO}$ and $\mathrm{OH}$ maser spectra (Fig. 1), but the CO feature may be of interstellar origin. In the case of two other objects, IRAS 18043-2116 and IRAS 18327-0715, we found relatively weak emission features that appear close to the expected central velocities, i.e. at $38 \mathrm{~km} \mathrm{~s}^{-1}$ and $87 \mathrm{~km} \mathrm{~s}^{-1}$ for IRAS 18043-2116 and IRAS 18327-0715, respectively (Fig. 1). These emission features are candidates for the circumstellar emission but their interstellar origin cannot be excluded without careful imaging observations and accurate positions. For these two sources, we obtained deep integrations in the $\mathrm{CO}(6-5)$ line (simultaneously with a spectrum in the $\mathrm{CO}(7-6)$ line). In these high rotational transitions, the contamination from interstellar clouds of low excitation should be negligible and the circumstellar gas can be identified more easily when excited to high $J$ levels. In both cases, the spectra do not show any emission in $\mathrm{CO}(6-5)$ nor in (7-6) at the noise levels given in Table 2. Our search for $\mathrm{CO}$ emission in these sources is therefore inconclusive.

In the case of $\mathrm{OH} 12.8-0.9$, the maser features appear in a velocity range that is not contaminated by strong interstellar $\mathrm{CO}$ emission. We detected weak and relatively broad emission features in $\mathrm{CO}(2-1)$ and (3-2) lines covering the same velocity range as the maser peaks of $\mathrm{H}_{2} \mathrm{O}$ and $\mathrm{OH}$, i.e. between -70 and $-35 \mathrm{~km} \mathrm{~s}^{-1}$ (Boboltz \& Marvel 2005). The detection is marginal, but the feature clearly appears above the $3 \sigma$ noise level after smoothing the spectra to a resolution of $5-6 \mathrm{~km} \mathrm{~s}^{-1}$, as can be seen in Fig. 2. That it appears in both lines and the good match with the maser velocities both support the detection, although more sensitive observations are desirable.
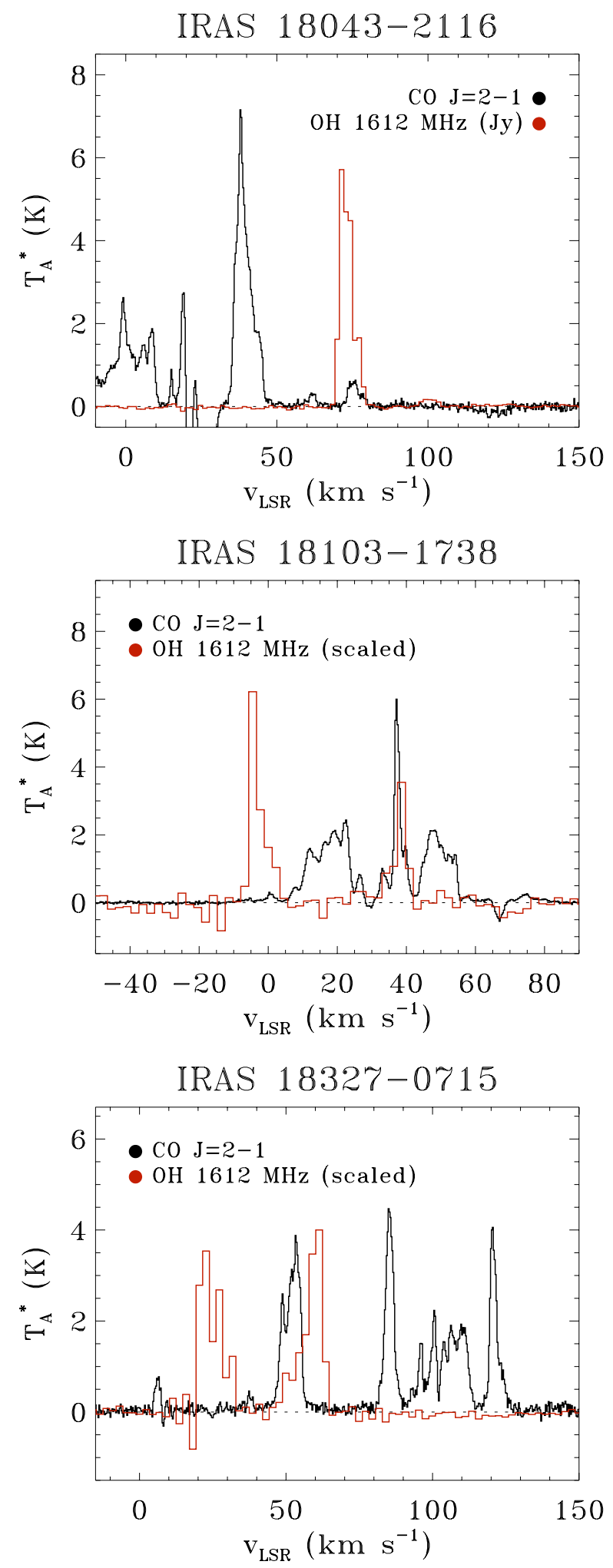

Fig. 1. Overlay of $\mathrm{OH}$ maser features on our $\mathrm{CO}$ spectra for targets with maser data available through on-line archives. The maser data are from Sevenster (2002) and available through VizieR (Ochsenbein et al. 2000, http://vizier.u-trasbg.fr/viz-bin/VizieR).

\section{IRAS 15452-5459: CO spectra}

We obtained three spectra corresponding to the central-star coordinates derived from observations performed with the Hubble 

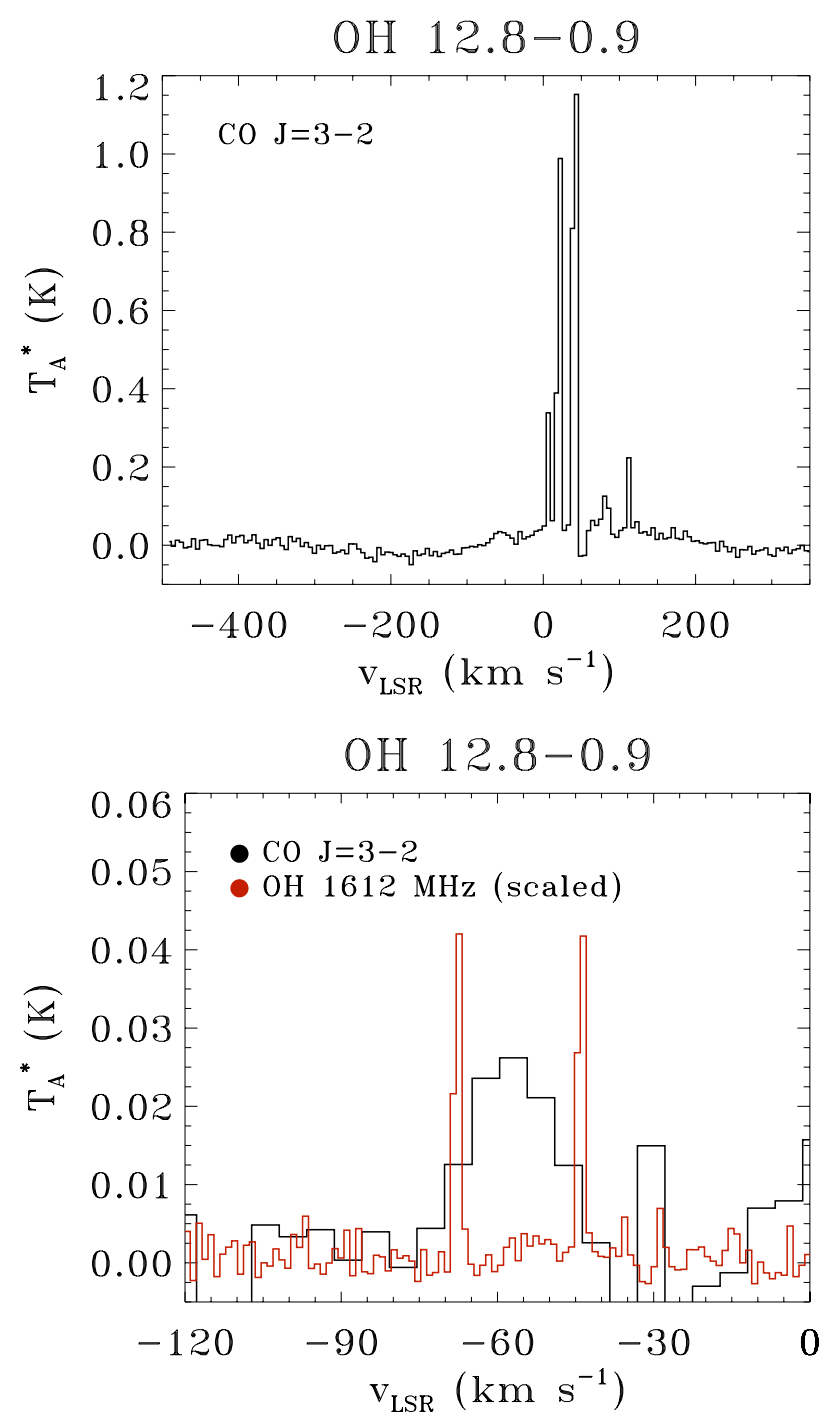

Fig. 2. $\mathrm{CO}$ spectrum observed toward $\mathrm{OH} 12.8-0.9$. In the bottom, the overlay of the smoothed spectrum and the $\mathrm{OH}$ maser spectrum is shown, as in Fig. 1.

Space Telescope (HST) and two positions that had been offset $7^{\prime \prime}$ from the central star position in both right ascension and declination. These are shown in Fig. 3.

The spectra corresponding to the central pointing appear as a superposition of at least two components: a broad feature and a narrow one. The broad feature seems to show an asymmetric profile, its red side being relatively abruptly cut off. Such a shape is likely due to an absorption feature around $-20 \mathrm{~km} \mathrm{~s}^{-1}$ clearly detected in the offset pointings, which is probably masking the line profile. The absorption was already noticed in the $\mathrm{OH}$ maser spectra by Deacon et al. (2004), who also pointed out that a similar feature is present around $-55 \mathrm{~km} \mathrm{~s}^{-1}$. These are likely spurious features due to the ISM emission in the off-source position. In Fig. 4, we compare the $\mathrm{CO}$ features with the $\mathrm{OH}$ maser spectrum published by Deacon et al. (2004) and note that the wide $\mathrm{CO}$ component extends over a much larger velocity range than the maser features. This broad component is not detected in the offset pointings, which indicates that the part of the outflow from which this feature originates is located in the inner region of the CSE. This is confirmed by the detection of the broad feature at all pointings in the $J=4-3$ line, which is a more robust tracer of the inner region than the $J=3-2$.

In the $J=3-2$ offset pointings, we detected only a central, strongly asymmetric double-horn component, whose red feature is much weaker than the blue one. At a close inspection, the spectral structure has more of a four-peak feature, resembling an optically thin, resolved double-shell.

\subsection{Kinematical components}

The data acquired northeast (NE) and southwest (SW) of the central star are characterized by a double-peak structure. The double-peak substructure of the blue peak is also easily seen in the SW pointing. An analogous substructure is probably responsible for the shape of the red peak. This closely resembles what is observed in the $\mathrm{OH}$ maser observations, where two features centered on $\sim-57 \mathrm{~km} \mathrm{~s}^{-1}$ also display multi-peak substructures.

By fitting Gaussians to the peaks, we derive for the NE feature a central velocity of $-57.5 \mathrm{~km} \mathrm{~s}^{-1}$ and a velocity width (FWHM) of $27.4 \mathrm{~km} \mathrm{~s}^{-1}$, while for the SW feature we obtain -55.5 and $28.6 \mathrm{~km} \mathrm{~s}^{-1}$, respectively.

As can be seen in Fig. 4, the narrow peaks match very well with the velocity ranges of the two central features observed in the $\mathrm{OH}$ maser spectrum by Deacon et al. (2004). They are also centered on the velocity accredited to the central star from the analysis of the $\mathrm{OH}$ maser lines. In spite of this, these narrow features cannot be conclusively distinguished from ISM contamination without carefully mapping the area around our target.

On the contrary, the broad component detected in the central pointing can certainly be attributed to I15452. We fit this component with a function of the form

$T_{\mathrm{mb}}(v)=T_{\mathrm{mb}}^{\text {peak }}\left[1-\left(\frac{V-V_{\mathrm{c}}}{V_{\mathrm{e}}}\right)^{2}\right],\left|V-V_{\mathrm{c}}\right|<V_{\mathrm{e}}$

and null elsewhere. As a central velocity $V_{\mathrm{c}}$, we assume $-57.5 \mathrm{~km} \mathrm{~s}^{-1}$ and find that $T_{\mathrm{mb}}^{\text {peak }} \sim 0.8 \mathrm{~K}$ and $V_{\mathrm{e}}=45.0 \mathrm{~km} \mathrm{~s}^{-1}$.

\subsection{Mass-loss rate}

We do not have observations of ${ }^{13} \mathrm{CO}$ lines that would allow us to estimate the optical depth of the ${ }^{12} \mathrm{CO}$ lines. We can derive the mass-loss rate associated with the outflow generating the broad component, from the correlation between this parameter and the $T_{\mathrm{mb}}$ at the center of the line.

For a spherically symmetric envelope, if we assume that its emission is optically thin, we can estimate the mass-loss rate from Knapp \& Morris (1985) and Oloffson et al. (1993) as

$\dot{M}=4.55 \times 10^{-19}\left[\frac{T_{\mathrm{mb}}}{\log (W / 0.04) s(J)}\right]^{5 / 6} f_{\mathrm{CO}}^{-1} V_{\exp }^{11 / 6}(D \theta)^{5 / 3}$,

where $T_{\mathrm{mb}}$ is the line peak main-beam temperature in $\mathrm{K}, W$ is the ratio of the flux emitted by the target at $4.6 \mu \mathrm{m}$ to that emitted by a star at the same distance with radius $5 \times 10^{13} \mathrm{~cm}$ approximated by a Planck curve at $2000 \mathrm{~K}, s(J)$ is a correction factor to account for the specific $J \rightarrow J-1$ transition, $f_{\mathrm{CO}}$ is the abundance of CO relative to $\mathrm{H}_{2}, V_{\text {exp }}$ the expansion velocity of the outflow in $\mathrm{km} \mathrm{s}^{-1}, \mathrm{D}$ the distance to the star in pc, and $\theta$ the beam size in $\operatorname{arcsec}$.

From our fitting to the component, we derive $T_{\mathrm{mb}}=0.8 \mathrm{~K}$ and $v_{\exp }=45 \mathrm{~km} \mathrm{~s}^{-1}$. To estimate the $W$ parameter, we perform synthetic photometry from the observed ISO spectrum of the source and calculate the IRAC $4.5 \mu \mathrm{m}$ flux density with the 

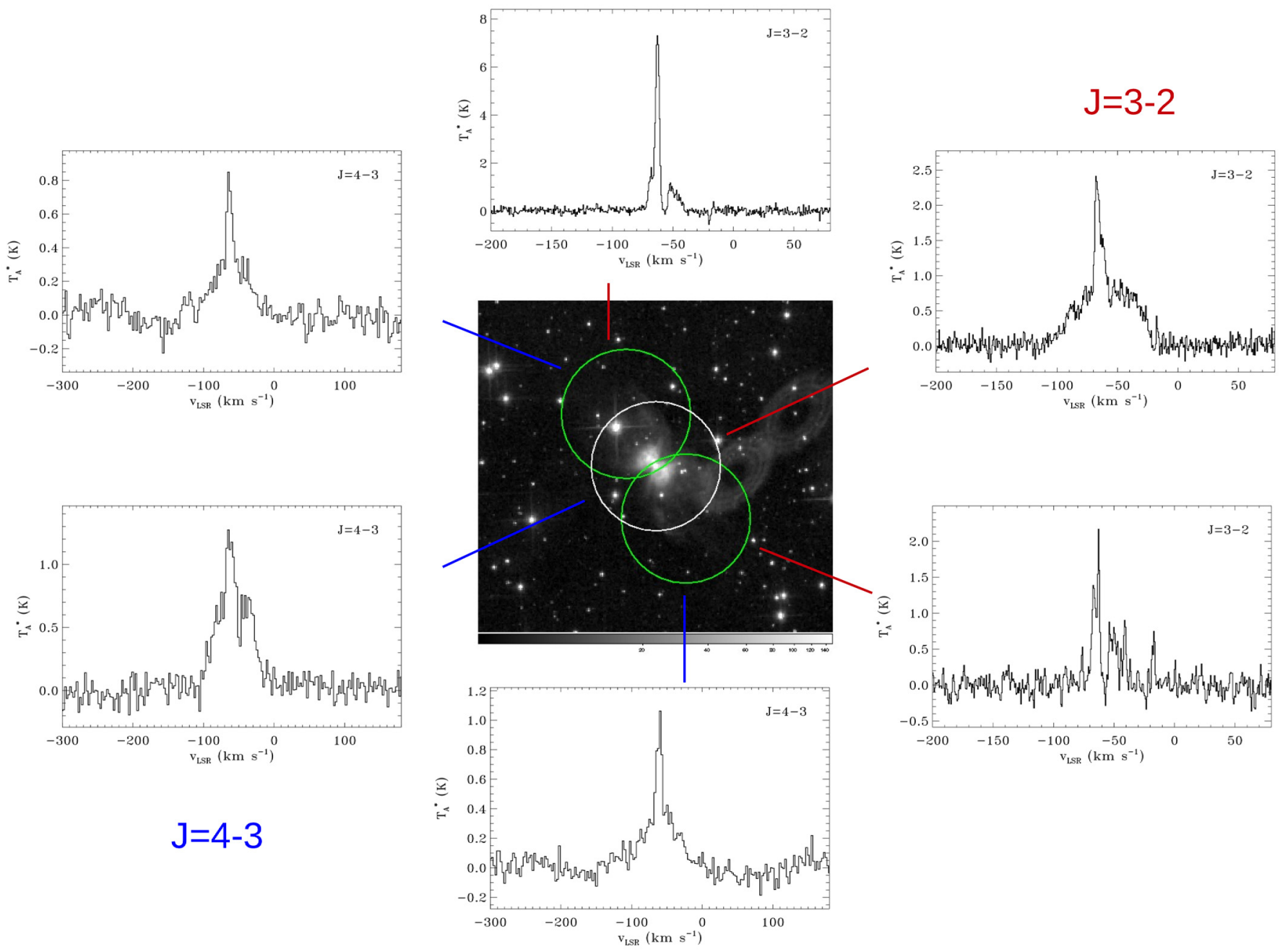

Fig. 3. Circles as wide as the APEX beam at $345 \mathrm{GHz}$ are centered on our pointing coordinates: a white circle indicates the central pointing and two green circles indicate the two pointings $10^{\prime \prime}$ offset. The $I$-band background image of I15452 was retrieved from the HST archive (north up and east left). Red bars indicate the spectra obtained for the $J=3-2 \mathrm{CO}$ line and blue bars those for the $J=4-3$ line.

Spitzer data reduction package SMART2 (Higdon et al. 2004), obtaining $11.38 \mathrm{Jy}$ (the source is included in the GLIMPSE survey (Churchwell et al. 2009), but its photometry at $4.5 \mu \mathrm{m}$ is unavailable from the on-line GLIMPSE catalog, probably because of saturation). We thus obtain $W=0.75$. The correction factor $s(3)$ is taken as 0.43 , following Groenewegen et al. (1999) and $f_{\mathrm{CO}}=3 \times 10^{-4}$, which is typical of O-rich circumstellar envelopes. Finally, the distance is set to $2.5 \mathrm{kpc}$ and the beam is $17.3^{\prime \prime}$. The derived mass-loss rate is $1.2 \times 10^{-4} M_{\odot} \mathrm{yr}^{-1}$.

Under the assumption of an optically thick line, the massloss rate can be estimated as (Groenewegen et al. 1999)

$\dot{M}=1.4 \frac{T_{\mathrm{mb}} V_{\mathrm{e}}^{2} D^{2} \theta^{2}}{2 \times 10^{19} f_{\mathrm{CO}}^{0.85} s(J)}$,

which returns a value of $4.9 \times 10^{-4} M_{\odot} \mathrm{yr}^{-1}$. In both cases, we obtain quite high values, but the calculations assume spherical symmetry, hence probably lead to overestimates.

By continuing to distinguish between the optically thin and thick regimes, we can calculate the mass of molecular gas corresponding to each spectral component. The $\mathrm{H}_{2}$ masses were

\footnotetext{
${ }^{2}$ SMART was developed by the IRS Team at Cornell University and is available through the Spitzer Science Center at Caltech.
}

derived by integrating over the whole spectrum the formula for the $i$ th channel

$M_{i}^{\mathrm{H}_{2}}=m_{\mathrm{H}_{2}} D^{2} \Omega N_{i}^{\mathrm{CO}} f_{\mathrm{CO}}$,

where the $\mathrm{CO}$ column density $N_{i}^{\mathrm{CO}}$ is given by Choi et al. (1993) as

$N_{i}^{\mathrm{CO}}=1.1 \times 10^{15} \frac{T_{\mathrm{mb}} \Delta v}{D\left(n, T_{K}\right)} \frac{\tau_{32}}{1-\exp \left(-\tau_{32}\right)}$.

$D\left(n, T_{K}\right)$ was shown to be equal to 1.5 to within a factor of 2 in the range $10<T_{K}<200 \mathrm{~K}$ and $10^{4}<n<10^{6} \mathrm{~cm}^{-3}, m_{\mathrm{H}_{2}}$ is the mass of a molecule of hydrogen, $D$ the distance to the star in $\mathrm{cm}$, and $\Omega$ the beam in sr. In Table 4 , we list values for $\tau_{32} \ll 1$ and for $\tau=5$ as a fiducial for the optically thick case, when indeed the mass depends on the real value of $\tau$. We also calculated the energy and momentum associated with the outflow along the line of sight by integrating $P_{i}=M_{i}\left|v_{i}-v_{0}\right|$ over the spectrum (the central velocity $v_{0}$ is $-57.5 \mathrm{~km} \mathrm{~s}^{-1}$ ) and then $E=\frac{P^{2}}{2 M}$.

The values of momentum and energy can be compared with those found by Bujarrabal et al. (2001). They found that most of the standard proto-PNe have outflows with kinetic energies in the range $10^{44}-10^{46} \mathrm{erg}$, with low-mass stars and hypergiants, 


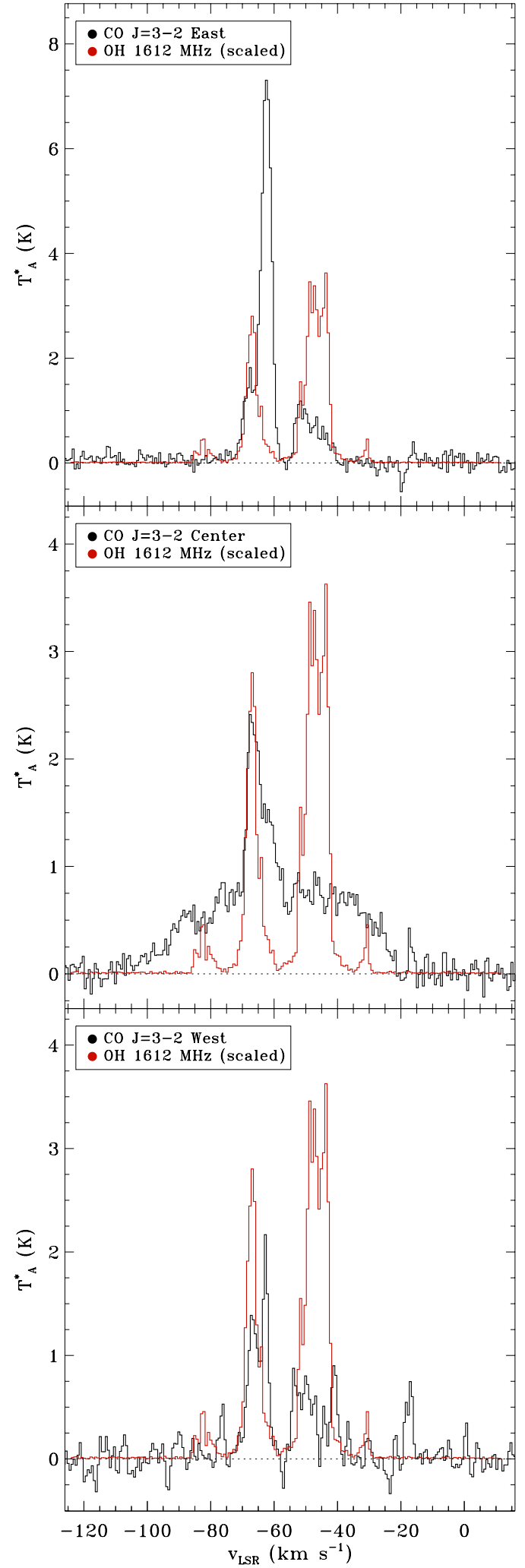

Fig. 4. Overlay of our CO $J=3-2$ data and $\mathrm{OH} 1612 \mathrm{MHz}$ (Deacon et al. 2004) lines for IRAS 15452-5459. The offsets from the central position in (RA, Dec) for the northeast and southwest pointings were $(7,7)$ and $(-7,-7)$, respectively.

respectively, below and above this range. Depending on the opacity of the features observed, we find in the molecular envelope around I15452 kinetic energies in the range $10^{42}-10^{44} \mathrm{erg}$, which would then imply its classification as a low-mass star.

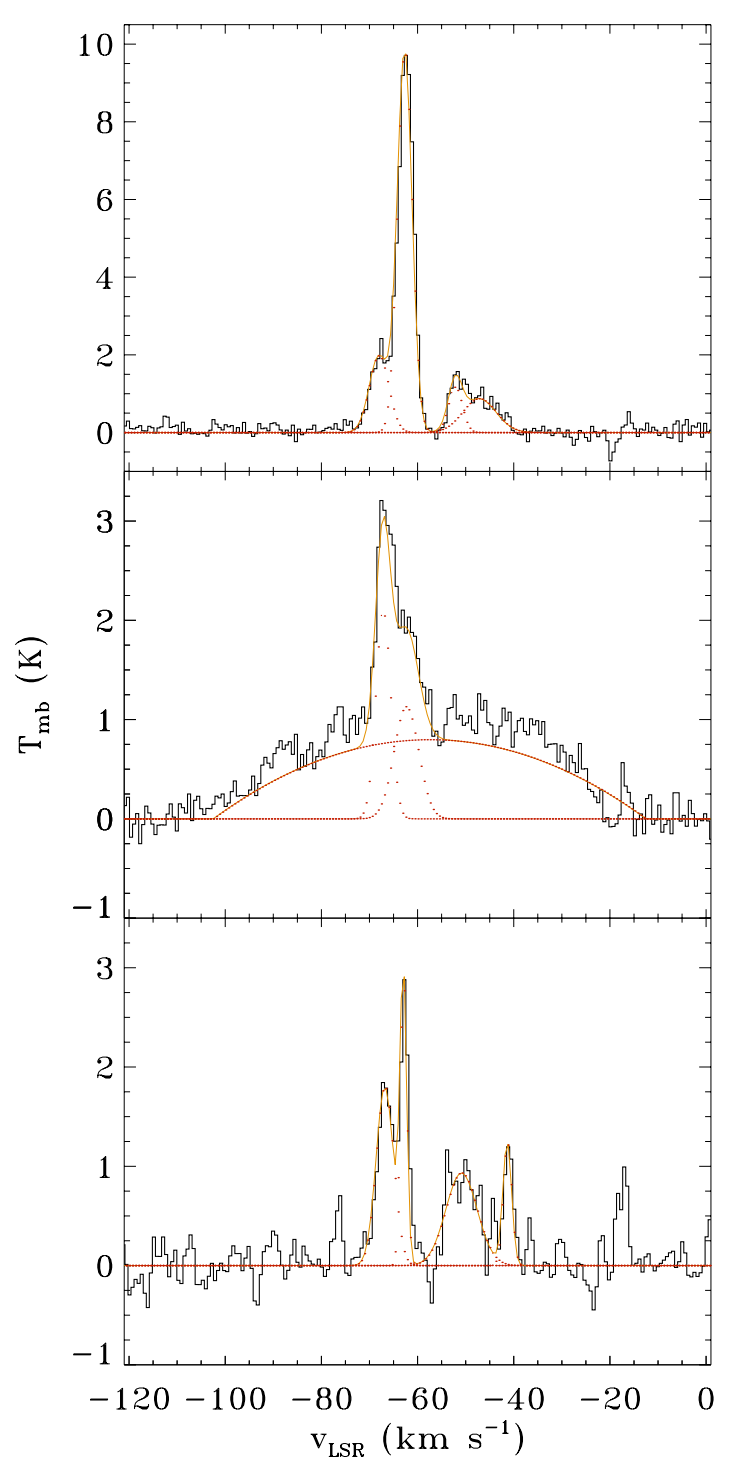

Fig. 5. Spectra of the $J=3-2$ line observed $10^{\prime \prime}$ northeast of the central source in IRAS 15452-5459 (top), on the central source (center) and $10^{\prime \prime}$ southwest of the central position (bottom). Overplotted in red are Gaussian fittings to the narrow features and a parabola for the broad component in the central pointing.

Bujarrabal et al. (2001) also find that if the origin of the outflow is radiation pressure on grains, an upper limit to the scalar momentum is given by $P \lesssim L / c\left(\mathrm{~g} \mathrm{~cm} \mathrm{~s}^{-1} \mathrm{yr}^{-1}\right) \times 2000(\mathrm{yr})$. For our source, $L / c=3.24 \times 10^{34} \mathrm{~g} \mathrm{~cm} \mathrm{~s}^{-1} \mathrm{yr}^{-1}$, therefore the upper limit to $P$ is $6.48 \times 10^{36} \mathrm{~g} \mathrm{~cm} \mathrm{~s}^{-1}$. We obtain for the momentum $6.96 \times 10^{36} \mathrm{~g} \mathrm{~cm} \mathrm{~s}^{-1}$ under the optically thin assumption. Even in this conservative case, the momentum is larger than can be accounted for by the radiation: the action of radiation on grains is unable to explain the outflow we observe in our target, as already found by Bujarrabal et al. (2001) for many proto-PNe.

\section{IRAS 15452-5459: IR archive observations}

The star was imaged with the HST at optical $(V)$ and near-IR ( $I$, $J, H$, and $K$ ) wavelengths by Sahai et al. (2007). The $V$-band images do not show any nebulosity and only the central star is weakly detected. The nebula becomes brighter at longer wavelengths, exhibiting an hourglass shape $\left(\sim 25^{\prime \prime}\right)$, as described by 


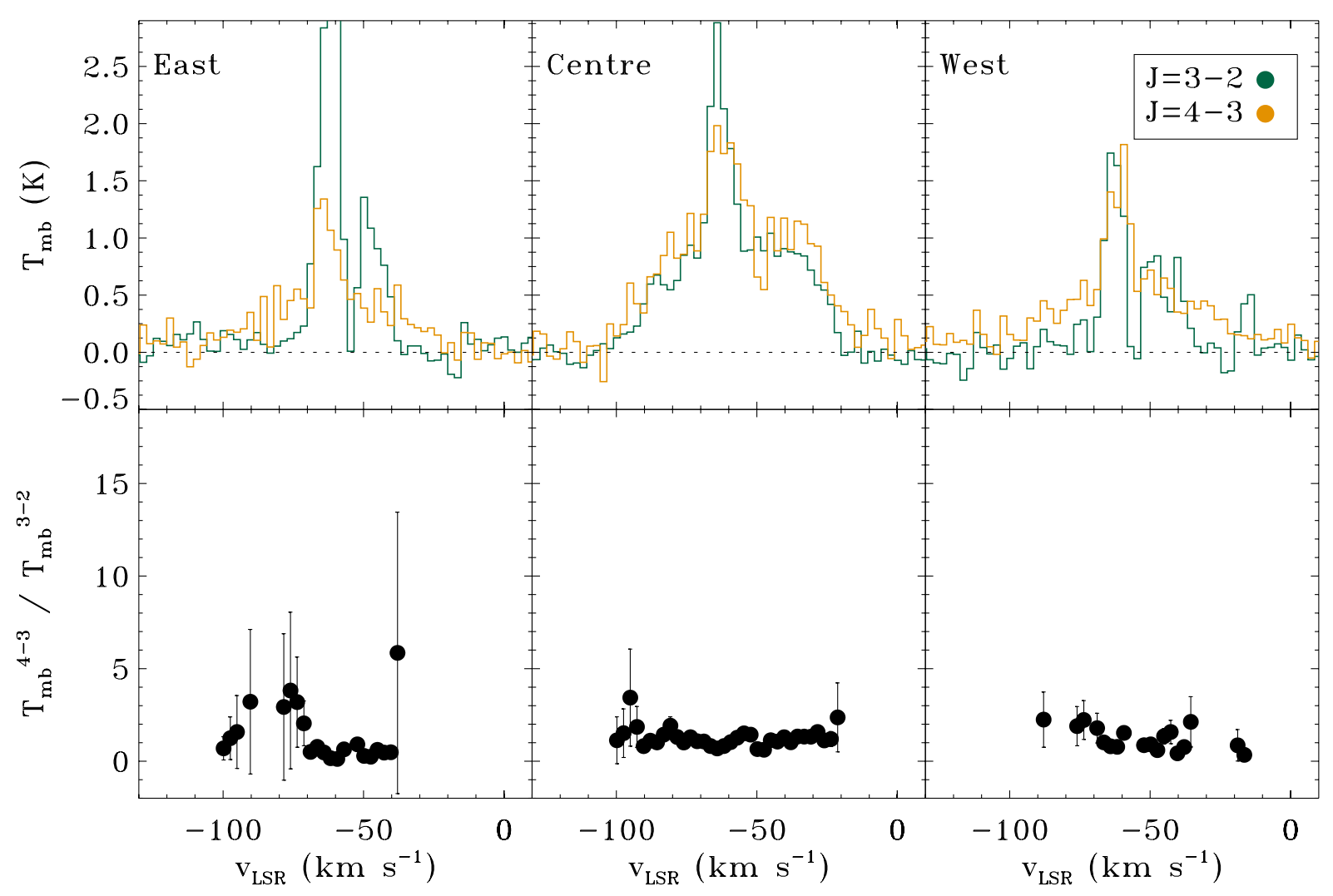

Fig. 6. Top: the two CO lines detected are plotted on each other, after resampling the spectra at a resolution of $2.5 \mathrm{~km} \mathrm{~s}^{-1}$. Bottom: the ratio of the two lines is shown for points where the emission from both lines is above their respective noises.

Table 4. Physical parameters derived for the outflow from the $J=$ 3-2 line.

\begin{tabular}{|c|c|c|c|}
\hline $\begin{array}{l}\dot{M} \\
10^{-4} M_{\odot} \mathrm{yr}^{-1}\end{array}$ & $\begin{array}{c}M_{\mathrm{H}_{2}} \\
10^{-3} M_{\odot}\end{array}$ & $\begin{array}{c}P \\
10^{36} \mathrm{~g} \mathrm{~cm} \mathrm{~s}^{-1}\end{array}$ & $\begin{array}{c}E \\
10^{42} \mathrm{erg}\end{array}$ \\
\hline \multicolumn{4}{|c|}{$\tau \ll 1$} \\
\hline 1.2 & 2.1 & 6.96 & 5.80 \\
\hline \multicolumn{4}{|c|}{$\tau=5$} \\
\hline 4.9 & 80 & 284 & 254 \\
\hline
\end{tabular}

Sahai et al. (2007), to which we refer for a morphological description.

\subsection{The ISO spectrum}

I15452 was observed with the Short and Long Wavelength Spectrometers on-board the Infrared Space Observatory (ISO). Its IR spectrum was presented by García-Lario et al. (2000), who reported the detection of the mid-IR features attributed to polycyclic aromatic hydrocarbons (PAHs) at 6.2, 7.7, and $8.6 \mu \mathrm{m}$, a plateau between 10 and $15 \mu \mathrm{m}$ also attributed to PAHs, and the $30 \mu \mathrm{m}$ feature due to $\mathrm{MgS}$. All of these features are typical of C-rich environments. The presence of both $\mathrm{PAH}$ features and the masers would make this object a member of the rare class of mixed-chemistry sources, whose nature is not well-understood. These may be stars that have changed their chemistry from O-rich into $\mathrm{C}$-rich and during the transition have the properties of both CSEs, or they may host structures that retain the O-rich material, despite the chemical change that occurs in the overall CSE. Another option is that photodissociation makes atomic $\mathrm{C}$ and $\mathrm{O}$ available for reaction and both O-bearing and C-bearing chemical species are then found in the CSE (Little-Marenin 1986).

The spectrum also contains several atomic forbidden emission lines of [OIII], [NIII], [NII], [CII], and [OI], which are typical of photodissociation regions (PDRs), but the continuum is clearly contaminated by spurious emission that can be attributed to Galactic cirrus. We retrieved the highly processed data set from the ISO web archive and in Table 5 we list the lines detected in the spectrum, their widths, and their fluxes. As can be seen in Fig. 7, the area where the source is located is rich in molecular gas and dust, and, since a background spectrum is unavailable, it cannot be ruled out that this diffuse gas affects or is responsible for the spectral features observed, keeping in mind that the FWHM of the ISO beam was $\sim 1.5^{\prime \prime}-93^{\prime \prime}$ in the $2-245 \mu \mathrm{m}$ range.

\subsection{PDR lines and circumstellar radiation field}

In a study of FIR PDR lines in AGB stars, proto-PNe, and PNe, Fong et al. (2001) and Castro-Carrizo et al. (2001) note that the detection of PDR lines depends mostly on the temperature of the central star, and that shocks, massive outflows, and the interstellar radiation field do not seem to play a role. On the basis of their observations, they distinguished circumstellar from interstellar PDRs by the flux ratio of the [CII] $(158 \mu \mathrm{m})$ to [OI] $(63 \mu \mathrm{m})$ lines. These authors observed that the former is always less intense than the latter, when both lines are clearly attributed to a circumstellar PDR. This clear attribution was impossible for their coldest sources, for which background spectra indicated 
Table 5. Atomic lines detected in the ISO spectrum of I15452.

\begin{tabular}{lccc}
\hline \hline Line & $\begin{array}{c}\text { Peak wavelength } \\
\mu \mathrm{m}\end{array}$ & $\begin{array}{c}\text { FWHM } \\
\mu \mathrm{m}\end{array}$ & $\begin{array}{c}\text { Flux } \\
10^{-18} \mathrm{~W} \mathrm{~cm}^{-2}\end{array}$ \\
\hline$[\mathrm{CII}](157.74)$ & $157.724 \pm 0.002$ & $0.662 \pm 0.002$ & $5.42 \pm 0.04$ \\
{$[\mathrm{OI}](145.52)$} & $145.54 \pm 0.03$ & $0.65 \pm 0.03$ & $0.16 \pm 0.02$ \\
{$[\mathrm{NII}](121.90)$} & $121.97 \pm 0.02$ & $0.99 \pm 0.02$ & $2.0 \pm 0.1$ \\
{$[\mathrm{OIII}](88.36)$} & $88.367 \pm 0.008$ & $0.316 \pm 0.007$ & $3.6 \pm 0.2$ \\
{$[\mathrm{OI}](63.18)$} & $63.191 \pm 0.007$ & $0.24 \pm 0.07$ & $0.79 \pm 0.07$ \\
{$[\mathrm{OIII}](51.81)$} & $51.79 \pm 0.02$ & $0.45 \pm 0.02$ & $3.9 \pm 0.6$ \\
{$[\mathrm{NIII}](57.33)$} & $57.32 \pm 0.01$ & $0.26 \pm 0.01$ & $1.7 \pm 0.2$ \\
{$[\mathrm{SiII}](34.84)$} & $34.793 \pm 0.003$ & $0.09 \pm 0.007$ & $0.43 \pm 0.03$ \\
{$[\mathrm{SIII}](33.48)$} & $33.46 \pm 0.01$ & $0.160 \pm 0.009$ & $2.4 \pm 0.4$ \\
\hline
\end{tabular}

Notes. In column one, the expected peak wavelengths are listed in $\mu \mathrm{m}$.

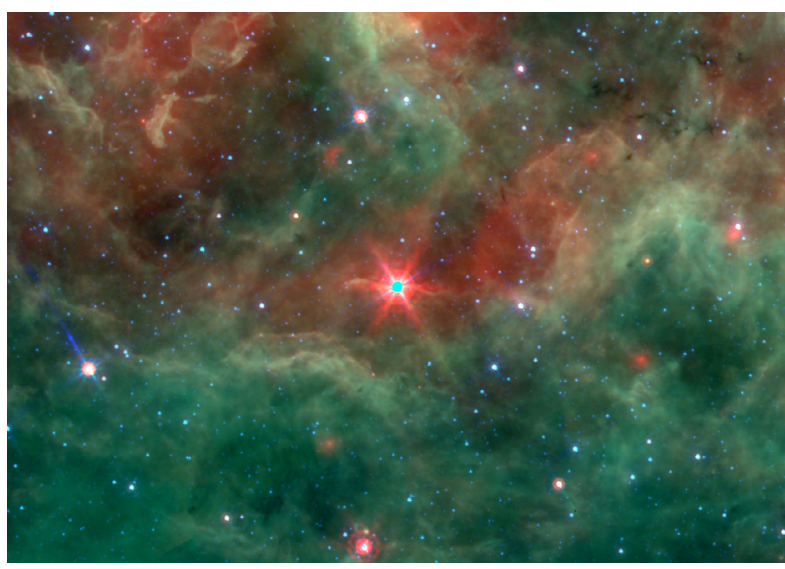

Fig. 7. Spitzer composite of the region around I15452 from the GLIMPSE and MIPSGAL archives. Images at 5.8, 8.0, and $24 \mu \mathrm{m}$ are coded on a logarithmic scale as blue, green, and red, respectively. The image covers an area about $24^{\prime} \times 19^{\prime}$ wide. North is up and east left.

interstellar contamination. This can bias the conclusion that in circumstellar PDRs $\mathrm{I}_{[\mathrm{CII}]}$ can be expected to be weaker than $\mathrm{I}_{[\mathrm{OI}]}$.

To characterize the average radiation field around the star, we can introduce the parameter $G$, the intensity of the UV radiation field in Habing units (Castro-Carrizo et al. 2001)

$G=\frac{L F_{\mathrm{UV}}}{4 \pi R_{i}^{2} G_{0}}$

where $L$ is the stellar luminosity, $F_{\mathrm{UV}}$ the fraction of energy at energies higher than $6 \mathrm{eV}(\sim 2066 \AA)$ in a blackbody spectrum at the same temperature as the star, $R_{i}$ is the inner radius from the star to the PDR, and $G_{0}=1.6 \times 10^{-3} \mathrm{erg} \mathrm{cm}^{-2} \mathrm{~s}^{-1}$ is the average UV flux in the diffuse ISM.

The ratio of the intensities of the [CII] $(158 \mu \mathrm{m})$ to [OI] $(63 \mu \mathrm{m})$ lines depends on the physical conditions in the PDR and the [CII] line is known to be the dominating coolant for average radiation fields with $G \lesssim 10^{3}$, while the [OI] dominates at higher values of $\mathrm{G}$. Therefore, although contamination is certainly possible, the atomic lines observed in the ISO spectrum of I15452 may indeed arise in a circumstellar PDR with a weak average radiation field.

We can calculate the expected value of $G$ for a star like I15452. The estimation of $R_{i}$ can be done by SED modeling, taking as $R_{i}$ the inner radius of the dust shell. To model the SED, we collected photometric data from several catalogs and used the code DUSTY (Ivezic et al. 1999) to solve the equation of

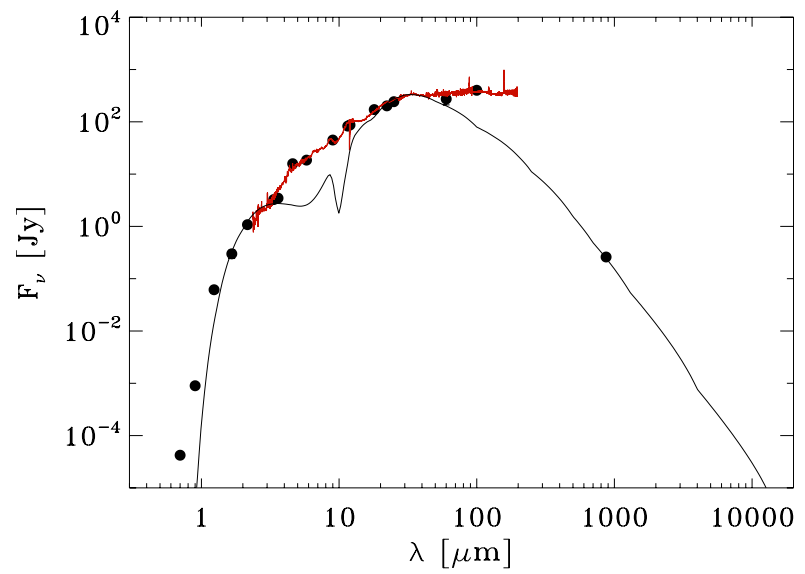

Fig. 8. SED of I15452 with the ISO spectrum shown in red and the DUSTY model as a black solid line. The data from Table 6 and our measurement at $345 \mathrm{GHz}(870 \mu \mathrm{m})$ are shown as solid circles.

radiation transfer in the circumstellar dust shell. DUSTY only considers spherical symmetry, which is clearly not our case, but it can still provide us with an approximate estimate of the inner radius. In Table 6, we listed the data collected from on-line catalogs, namely USNOB1, 2MASS, GLIMPSE, WISE, AKARI, and IRAS, which are shown in Fig. 8.

We ran our model with $T_{\star}=3500 \mathrm{~K}$, standard density distribution of $r^{-2}$, grain size distribution with a minimum radius $a_{\min }=0.005$ and a maximum one $a_{\max }=0.25 \mu \mathrm{m}$ varying as $a^{-0.35}$ and optical constants for amorphous silicates (appropriate for O-rich environments). With the exception of the mid-IR range, where the excess of emission can be regarded as an indication of a disk, a good fit to the observational data could be obtained for a distance of $2.5 \mathrm{kpc}$, a luminosity of $8000 L_{\odot}$, and a circumstellar absorption $A_{\mathrm{V}}=58(\tau \sim 1$ around $3.35 \mu \mathrm{m})$.

From our DUSTY modeling, we can estimate a FIR continuum flux of $1.7 \times 10^{-11} \mathrm{~W} \mathrm{~m}^{-2}$ and an inner radius of $5.7 \times$ $10^{15} \mathrm{~cm}$. Therefore, we find $G \sim 170$, with $F_{\mathrm{UV}}=3.4 \times 10^{-6}$, where I15452 seems then to fall within the regime where the [CII] line is the dominant coolant.

To estimate the intensity of the FUV field and the density in the PDR, we use the models described in Kaufman et al. (2006) ${ }^{3}$. For a given set of gas-phase elemental abundances and grain properties, each model is described by a constant $\mathrm{H}$ nucleus

\footnotetext{
3 The models are available on-line at http: //dustem. astro.umd. edu/.
} 
Table 6. Photometric data on I15452 collected from on-line catalogs.

\begin{tabular}{llcccc}
\hline \hline \multirow{2}{*}{ USNOB1 } & $\lambda[\mu \mathrm{m}]$ & 0.7 & 0.9 & & \\
& $F_{\lambda}[\mathrm{mJy}]$ & $0.042 \pm 0.004$ & $0.90 \pm 0.09$ & & \\
\multirow{2}{*}{2 MASS } & $\lambda[\mu \mathrm{m}]$ & 1.235 & 1.662 & 2.159 & \\
& $F_{\lambda}[\mathrm{mJy}]$ & $61.5 \pm 8.7$ & $299 \pm 56$ & $1080 \pm 30$ & \\
\hline \multirow{2}{*}{ IRAC } & $\lambda[\mu \mathrm{m}]$ & 3.6 & 5.8 & & \\
& $F_{\lambda}[\mathrm{Jy}]$ & $3.470 \pm 0.49$ & $18.52 \pm 0.76$ & & 22.24 \\
\multirow{2}{*}{ WISE } & $\lambda[\mu \mathrm{m}]$ & 3.35 & 4.6 & 11.56 & $201.8 \pm 3.7$ \\
& $F_{\lambda}[\mathrm{Jy}]$ & $3.26 \pm 0.37$ & $15.81 \pm 0.73$ & $82.77 \pm 0.61$ & \\
\multirow{2}{*}{ AKARI } & $\lambda_{[}[\mu \mathrm{m}]$ & 9.0 & 18.0 & & 100 \\
& $F_{\lambda}[\mathrm{Jy}]$ & $44.8 \pm 3.9$ & $171.4 \pm 10.7$ & & 401 \\
\hline \multirow{2}{*}{ IRAS } & $\lambda[\mu \mathrm{m}]$ & 12 & 25 & 60 & \\
& $F_{\lambda}[\mathrm{Jy}]$ & $87 \pm 9$ & $243 \pm 12$ & $274 \pm 63$ & \\
\hline
\end{tabular}

Notes. Infrared data are available on line through the IRSA archive (http://irsa.ipac. caltech.edu), while optical data are available through VizieR (Ochsenbein et al. 2000, http://vizier.u-trasbg.fr/viz-bin/VizieR).

density, $n$, and incident far-ultraviolet intensity $G$. The models solve for the equilibrium chemistry, thermal balance, and radiation transfer through a PDR layer. The values of $n_{\mathrm{H}}$, and $G$ are obtained by overlaying two sets of contour plots and finding the intersection of the observations. The procedure requires either two sets of line-intensity ratios or one set of line-intensity ratios and the ratio of the $[\mathrm{CII}]+[\mathrm{OI}]$ intensity to that of the FIR dust continuum emission. The abundances used in the calculation were $[\mathrm{C} / \mathrm{H}]=1.4 \times 10^{-4},[\mathrm{O} / \mathrm{H}]=3 \times 10^{-4},[\mathrm{Si} / \mathrm{H}]=1.7 \times 10^{-6}$, $[\mathrm{S} / \mathrm{H}]=2.8 \times 10^{-5},[\mathrm{Fe} / \mathrm{H}]=1.7 \times 10^{-7},[\mathrm{Mg} / \mathrm{H}]=1.1 \times 10^{-6}$, and $[\mathrm{PAH} / \mathrm{H}]=4 \times 10^{-7}$.

Either a filling-factor correction should be applied to each line or line intensities should be used, as lines of different species do not necessarily arise from the same regions. For example, diffuse [CII] emission may fill the observing beam, whereas [OI] emission may arise from a smaller region of a higher density and temperature. A similar correction should also be applied to the FIR continuum.

In Fig. 9, we show contour plots for the ratios of the sum of the two PDR lines to the FIR continuum as a function of $G$ and $n$, obtained from the PDR modeling. In the rough assumption that all of the emitting components arise from regions with similar sizes, we have $\left(I_{[\mathrm{OI}]}+I_{[\mathrm{CII}]}\right) / I_{\mathrm{FIR}} \sim 0.0037$. For our calculated value of $G$, this corresponds in Fig. 9 to a density of $117 \mathrm{~cm}^{-3}$. This density value is somewhat low for a post-AGB CSE, but may be found in the tenuous region where the fast wind has swept away most of the material.

\subsection{Envelope mass}

We can derive the mass of dust by simply fitting a modified blackbody to the observational data beyond $20 \mu \mathrm{m}$, neglecting the IRAS point at $100 \mu \mathrm{m}$, because it is clearly contaminated. This procedure provides us with an average dust temperature $T_{\mathrm{d}}$ and a dust emissivity index $\beta$ that can be used to calculate the mass of the dust following Beuther et al (2005)

$M_{\text {dust }}=\frac{2.0 \times 10^{-4}}{J_{v}\left(T_{\mathrm{d}}\right)} \frac{F_{v}}{\mathrm{Jy}}\left(\frac{D}{\mathrm{kpc}}\right)^{2}\left(\frac{v}{1.2 \mathrm{THz}}\right)^{-3-\beta} M_{\odot}$

$J_{v}\left(T_{\mathrm{d}}\right)=\left[\exp \left(\frac{h v}{k T_{\mathrm{d}}}\right)-1\right]^{-1}$

A15, page 10 of 12

$$
([\mathrm{OI}] 63 \mu \mathrm{m}+[\mathrm{CII}] 158 \mu \mathrm{m}) / \mathrm{I}_{\mathrm{IR}}
$$

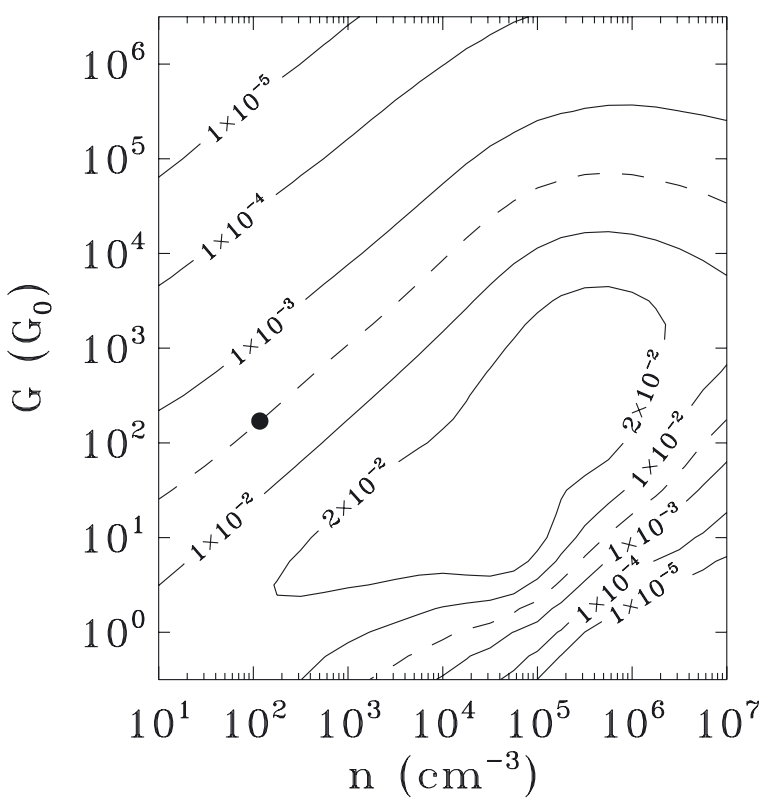

Fig. 9. Contour plots of the ratio of the intensity of the [CII] line plus that of the $[\mathrm{OI}]$ line over the FIR continuum as a function of the intensity of the FUV field $G$ and the atomic $\mathrm{H}$ density $n$. The dashed contour corresponds to the value of 0.0037 found for I15452 and the solid circle corresponds to $G=170$ and $n=117$.

Eq. (7) assumes an average grain size of $0.1 \mu \mathrm{m}$ and a grain density of $3 \mathrm{~g} \mathrm{~cm}^{-3}$, where $h$ and $k$ are the Planck and Boltzmann constants, D is the distance to the star $(2.5 \mathrm{kpc})$, and $F_{v}$ the flux density measured at the frequency $v$, which in our case is that of our LABOCA measurement, $345 \mathrm{GHz}$.

The fit to the FIR/sub-mm observational points gives us values of $T_{\mathrm{d}} \sim 95 \pm 9 \mathrm{~K}$ and $\beta \sim 1.10 \pm 0.14$, which allows us to calculate a CSE dust mass of $0.010 \pm 0.003 M_{\odot}$. The uncertainties listed are the statistical errors resulting from the fitting procedure and the error propagation in Eq. (7).

Castro-Carrizo et al. (2001) derived a formula to estimate the mass of atomic gas from the flux observed in the [CII] line at $157.8 \mu \mathrm{m}$, which we report to be

$M\left(M_{\odot}\right)=7.0 \times 10^{6} F_{[\mathrm{CII}]}\left(\mathrm{erg} \mathrm{cm}^{-2} \mathrm{~s}^{-1}\right) D(\mathrm{kpc})^{2} F_{\mathrm{c}} / X_{\mathrm{C}}$, 
where $F_{[\mathrm{CII}]}$ is the observed flux in the [CII] line, $D$ the distance to the star, $X_{\mathrm{C}}$ the abundance of $\mathrm{C}\left(1.5 \times 10^{-3}\right.$ for C-rich stars and $2 \times 10^{-4}$ for O-rich sources), and $F_{\mathrm{c}}$ is a correction factor that accounts for low excitation temperatures. The correction factor is always $\geq 1$ and depends on the density and kinetic temperature of the gas, although at the densities found in proto-PNe it is unlikely to be larger than two or three.

From Eq. (8), we can then calculate the mass of atomic gas in the nebula around I15452 to be about $1.9 M_{\odot} D(\mathrm{kpc})^{2}$, assuming $F_{\mathrm{c}}=1$. We note that I15452 appears to be surrounded by a massive CSE, which still assuming $F_{\mathrm{c}}=1$, would contain about $12 M_{\odot}$ of gas, at the distance of $2.5 \mathrm{kpc}$. A statistical error in the mass estimate may be calculated from the error in the flux of the [CII] line, but this would not account for the real uncertainty in the mass, which is much larger and due to the degree of contamination. The high value of mass found is beyond those expected from intermediate-mass stars and contrasts with the small momentum found above for the molecular component, which is considered as an indication that the source is a lowmass star. Such a high estimate of the circumstellar atomic mass can be regarded as an indication of the ISM contamination of the FIR spectrum.

For a gas-to-dust mass ratio of 200 - a standard value in evolved stars - our dust mass estimate implies a gas mass of about $2 M_{\odot}$. Contamination would then enhance the flux in the [CII] line by a factor of six.

\section{Discussion and conclusions}

We have observed a sample of stars where the detection of maser velocities larger than observed on the AGB indicates that these sources have started their post-AGB evolution. In particular, we have included four of the water-fountain nebulae known that were not previously investigated for $\mathrm{CO}$ emission. As our targets are located in the Galactic plane, spurious emission from the interstellar medium typically contaminates the spectra and does not allow us to obtain conclusive detections. By comparing the maser features with the $\mathrm{CO}$ lines, we find hints of possible $\mathrm{CO}$ detections, but only with sensitive maps of the regions around the targets can a distinction between ISM and circumstellar CO be made.

Only IRAS $15452-5459$ is clearly detected in both the $J=$ $3-2$ and $J=4-3$ lines. The star shows in its central region a fast CO outflow with velocities of about $V_{\exp }=45 \mathrm{~km} \mathrm{~s}^{-1}$, while narrow peaks are indicative of an expansion velocity of $\sim 14 \mathrm{~km} \mathrm{~s}^{-1}$. The latter falls within the range of velocities observed during the AGB and, as it is detected at all pointings, it can be interpreted as the result of the remnant AGB wind. With its four peaks, the $\mathrm{CO}$ feature resembles those observed in the $\mathrm{OH}$ maser and points to a complex emitting structure, rather than an expanding spherical envelope. The broad component observed only in the central region of the source points instead to an outflow of molecular gas. Only interferometric observations can estimate whether this outflow is in the form of a collimated wind. The mass-loss rate calculated for this component is quite high $\left(\sim 10^{-4} M_{\odot} \mathrm{yr}^{-1}\right)$ but the assumed spherical symmetry implies that the value is likely overestimated. We have also calculated the scalar momentum and kinetic energy associated with the outflowing material, which we have compared with the results obtained by Bujarrabal et al. (2001), concluding that radiation pressure on dust grains alone cannot explain the dynamics of the CSE.

By fitting a modified blackbody to the FIR/sub-mm observational points of I15452, we have estimated a dust mass in its CSE of $\sim 0.01 M_{\odot}$, while through the analysis of its ISO spectrum, we have calculated an envelope gas mass of about $12 M_{\odot}$. The uncertainty in the latter value is dominated by the degree of contamination of the [CII] line. The derived values of gas and dust masses (12 and $2 M_{\odot}$, respectively) indicate an enhancement factor of six in the line flux because of contamination, if we assume a standard value of 200 for the gas-to-dust mass ratio.

Nevertheless, the total CSE mass of I15452 may be higher than $2 M_{\odot}$ and the gas-to-dust mass ratio might also be higher than its standard value. 115452 belongs to a group of $\mathrm{OH}$ masing stars classified by Sevenster (2002) as LI, because they lie on the left of the evolutionary sequence on the IRAS color-color diagram, while "standard" post-AGB stars lie on the right side (RI sources). LI stars have higher outflow velocities and massloss rates and they may loop back to the left of the evolutionary sequence because of an early AGB termination, which would also cause them to host $\mathrm{OH}$ masers for a much longer time than RI stars (Sevenster 2002). Finally, by scale height considerations, Sevenster (2002) argue that LI stars may be the precursors of bipolar planetary nebulae and have higher masses than RI sources (ending in elliptical planetary nebulae), with average values of 4 and $1.7 M_{\odot}$ for LI and RI, respectively (Deacon et al. 2007). The bipolarity of the nebula around 115452 has indeed been proven by Hubble images. For this star to have a CSE mass within values adequate for LI sources (a minimum of 3-4 $M_{\odot}$ ), its gas-to-dust mass ratio must be at least 300-400, based on our dust mass estimate. This would decrease the degree of contamination of the [CII] line mentioned above to a factor of 3-4.

I15452 is certainly a peculiar object from many points of view. For example, the four peaks seen in its satellite $\mathrm{OH}$ line are almost unique among the several hundreds of evolved stars where this feature has been detected. Another target with such a profile of its $\mathrm{OH}$ maser is $\mathrm{OH} 19.2-1.0$, in which the spectral shape of the features shows a smaller degree of symmetry than that in I15452. The four peaks in I15452 are stunningly similar to one of the profiles derived theoretically by Grinin \& Grigor'ev (1983) for an unsaturated maser originating from a rotating and expanding disk. As pointed out by Chapman (1988) for OH 19.2-0.1, the applicability of the calculations by Grinin \& Grigor'ev (1983) to an evolved star is dubious, because $\mathrm{OH}$ masers in these sources are typically saturated. Nevertheless, the mid-IR excess of its SED and the mixed chemistry may actually point to the existence of a disk in I15452. The detection of the PAH features in a region of the ISO spectrum that does not appear to be affected by ISM contamination and the presence of O-bearing molecules can in fact be explained by a circumstellar disk. This disk would serve as a reservoir of O-rich material, where the masers would arise, while the PAHs would be located in the circumstellar envelope, which would be converted into C-rich material by the third dredge-up.

We would require a high angular-resolution mid-IR spectrum, in addition to high angular-resolution observations of both the masers and the $\mathrm{CO}$ outflow to reconstruct the morphology of the central emitting region in $\mathrm{I} 15452$.

Acknowledgements. We would like to thank our referee, Dr Jessica Chapman, for comments that helped to improve this paper. This research has made use of the VizieR catalog access tool, CDS, Strasbourg, France. This research has made use of the NASA/ IPAC Infrared Science Archive, which is operated by the Jet Propulsion Laboratory, California Institute of Technology, under contract with the National Aeronautics and Space Administration.

\section{References}

Beuther, H., Schilke, P., Menten, K. M., et al. 2005, ApJ, 633, 535 Boboltz, D. A., \& Marvel, K. B. 2005, ApJ, 627, L45 
Bujarrabal, V., Castro-Carrizo, A., Alcolea, J., \& Sánchez-Contreras, C. 2001, A\&A, 377, 868

Carey, S., Noriega-Crespo, A., Mizuno, D. R., et al. 2009, PASP, 121, 76

Castro-Carrizo, A., Bujarrabal, V., Fong, D., et al. 2001, A\&A, 367, 674

Cerrigone, L., Hora, J. L., Umana, G., \& Trigilio, C. 2009, ApJ, 703, 585

Chapman, J. 1988, MNRAS, 230, 415

Choi, M., Evans, N. J., \& Jaffe, D. T. 1993, ApJ, 417, 624

Churchwell, Ed, Babler, Brian L., Meade, Marilyn R., et al. 2009, PASP, 121, 213

Day, F. M., Pihlströ, Y. M., Claussen, M. J., \& Sahai, R. 2010, ApJ, 713, 986

Deacon R. M., Chapman, J. M., \& Green, A. J. 2004, ApJS, 155, 595

Deacon R. M., Chapman, J. M., Green, A. J., \& Sevenster, M. N. 2007, ApJ, 658, 1096

Engels, D. 2002, A\&A, 388, 252

Fong, D., Meixner, M., Castro-Carrizo, A., et al. 2001, A\&A, 367, 652

García-Lario, P., Manchado Torres, A., Ulla, A., Manteiga, M., \& Suárez Fernandez, O. 2000 ASPC, 199, 391

Gómez, Y., Rodríguez, L. F., Contreras, M. E., \& Moran, J. M. 1994, RMA\&A, 28,97

Grinin, V. P., \& Grigor'ev, S. A. 1983, Astron. Zh., 60, 512

Groenewegen, M. A. T., Baas, F., Blommaert, J. A. D. L., et al. 1999, A\&AS, 140,197

Güsten, R., Nyman, L. Å., Schilke, P., et al. 2006 A\&A, 454, L13

Habing, H. J. 1996, A\&ARv, 7, 97

He, J. H., Imai, H., Hasegawa, T. I., Campbell, S. W., \& Nakashima, J. 2008, A\&A, 488, L21

Heyminck, S., Kasemann, C., Güsten, R., de Lange, G., \& Graf, U. U. 2006, A\&A, 454, L21

Higdon, S. J. U., Devost, D., Higdon, J. L., et al. 2004, PASP, 116, 975
Imai, H., He, J.-H., Nakashima, J.-I., et al. 2009, PASJ, 61, 1365

Ivezic, Z., Nenkova, M., \& Elitzur, M. 1999, User Manual for DUSTY, University of Kentucky Internal Report, accessible at http://www.pa.uky.edu/ moshe/dusty

Kasemann, C., Güsten, R., Heyminck, S., et al. 2006, in SPIE Conf. Ser., 6275, $62750 \mathrm{~N}$

Kaufman, M. J., Wolfire, M. G., \& Hollenbach, D. J. 2006, ApJ, 644, 283

Klein, B., Philipp, S. D., Krämer, I., et al. 2006, A\&A, 454, L29

Knapp, G. M., \& Morris, M. 1985, ApJ, 292, 640

Likkel, L., \& Morris, M. 1988, ApJ, 329, 914

te Lintel Hekkert, P., Caswell, J. L., Habing, H. J., et al. 1991, A\&AS, 90, 327

Little-Marenin, I., R. 1986, ApJ, 307, L15

Ochsenbein, F., Bauer, P., \& Marcout, J. 2000, A\&AS, 143, 221

Olofsson, H., Eriksson, K., Gustafsson, B., \& Carlstrom, U. 1993, ApJS, 87, 267

Oudmaijer, R. D., Waters, L. B. F. M., van der Veen, W. E. C. J., \& Geballe, T. R. 1995, A\&A, 299, 690

Pardo, J. R., Cernicharo, J., \& Serabyn, E. 2001, IEEE Trans. Antennas Propag., 49,1683

Ramstedt, S., Schöier, F. L., Olofsson, H., \& Lundgren, A. A. 2008, A\&A, 487, 645

Risacher, C., Vassilev, V., Monje, R., et al. 2006, A\&A, 454, L17

Sahai, R., Morris, M., Sánchez Contreras, C., \& Claussen, M. 2007, AJ, 134, 2200

Sevenster, M. N. 2002, AJ, 123, 2772

Siringo, G., Kreysa, E., Kovács, A., et al. 2009, A\&A, 497, 945

Urquhart, J. S., Busfield, A. L., Hoare, M. G., et al. 2007, A\&A, 474, 891

van der Veen, W. E. C. J., \& Habing, H. J. 1988, A\&A, 194, 125

van der Veen, W. E. C. J., Habing, H. J., \& Geballe, T. R. 1989, A\&A, 226, 108

Werner, M. W., Roellig, T. L., Low, F. J., et al. 2004, ApJS, 154, 1 